\title{
Análisis Del Comercio De Aguacate De La Unión Europea Con México A Partir Del Acuerdo De Asociación; Comparación Con El Comercio De Chile Y La Unión Europea Del Período 2001 Al 2016
}

\author{
M.C. Carlos Ernesto Luquez, Gaitán (Doctorante) \\ UACh, México \\ Dra. Alma Alicia Gómez
}

Profesor Investigador de Universidad Autónoma Chapingo, México

Dr. Stéphan Sberro Picard

Profesor Investigador de Instituo Tecnológico Autónomo de México,

Ciudad de México, CDMX

\section{Doi:10.19044/esj.2018.v14n25p335 URL:http://dx.doi.org/10.19044/esj.2018.v14n25p335}

\begin{abstract}
This paper focuses on analyzing the bilateral aspects regarding avocado; the elimination of import tariffs from the European Union from Chile and Mexico; and the terms under which export tariffs are released. In the middle of the first semester of 2018, Mexico and the European Union negotiated the modernization of the Agreement. The activities that are most beneficial to the association agreement in Mexico are those of the primary sector. The exported volumes, negotiated prices, and the market shares between Mexico and the European Union were collected. The objective of this article is to compare the commercial exchange between the European Union, Chile, and Mexico. It aims to analyze the Chile-European Community Association Agreement, so as to draw conclusions on the evolution of the avocado trade generated from the agreement. It also compares the commercial experience of Chile with the European Union and the experience of Mexico and the European Union. The analysis involves the collection of statistical information in order to appreciate the evolution of exports from Chile to the European Union; exports of avocado from Mexico to the European Union in the period between 2001 and 2016; and the generation of indicators such as the price per average annual ton, export growth rates, and proportions in the European market. The main result shows that despite a similar agreement between both competitors and the European Union, Chile is the country that has experienced the most growth in exports and has a greater market share.
\end{abstract}


Keywords: Tax relief, tariffs, prices, market shares

\section{Resumen}

Este artículo analiza los aspectos bilaterales en materia de aguacate; la eliminación de aranceles para importación desde la Unión Europea desde Chile y México; los términos bajo el cual se libera de aranceles a la exportación. A mediados del primer semestre de año 2018, México y la Unión Europea negocian la modernización del Acuerdo. Las actividades más beneficiadas por el acuerdo de asociación en México son las del sector primario. Se recopilan los volúmenes exportados, precios negociados, cuotas de mercado entre México con la Unión Europea. Los objetivos del artículo son comparar el intercambio comercial entre Unión Europea, Chile y México; analizar el Acuerdo de Asociación Chile-Comunidad Europea, para hacer conclusiones de la evolución del comercio de aguacate generado a partir del acuerdo. También comparar la experiencia comercial de Chile con la Unión Europea y la experiencia de México y con la Unión Europea. El análisis, implica la recopilación de información estadística con el fín de apreciar la evolución de las exportaciones de Chile a la Unión Europea, y las exportaciones de México a la Unión Europea, de aguacate, en el periodo entre 2001 y 2016, y la generación de indicadores como el precio por tonelada promedio anual, tasas de crecimiento de la exportaciones y proporciones en el mercado europeo. El principal resultado es que a pesar de un similar acuerdo entre ambos competidores y la Unión Europea; Chile es el país que más crecimiento en las exportaciones ha experimentado y posee mayor cuota de mercado.

Palabras claves: Desgravación, aranceles, precios, cuotas de mercado.

\section{Introducción}

Los Acuerdos de Asociación, tratados de libre comercio u otros instrumentos están enfocados en potenciar el libr cambio de mercancías, surgen generalmente de los países que tienen precedentes comerciales entre ellos y optan por formalizar las alianzas con acuerdos internacionales y, esto trae consecuencias negativas o positivas para cada una de las partes asociadas visto desde el punto de vista de las ventajas comparativa; teoría planteada ya hace mucho tiempo por David Ricardo en una Inglaterra principal protagonista del avance económico.

La Unión Europea se ha consolidado como uno de los principales bloques comerciales a nivel mundial; han logrado avances únicos en consolidación comunitaria, lo que se interpretara para los fines del análisis, como una fuerza comercial unificada con un mismo mercado e intereses, aunque se traten de distintas naciones. 
México fue el primer país de América Latina en establecer un acuerdo de asociación con la Unión Europea. En marzo de 2000 se firmó el Tratado de Libre Comercio entre la UE y México que entró en vigor el primero de julio del mismo año; a raíz de este tratado México se convirtió en el país latinoamericano con la canasta más diversificada de productos exportados a la Unión Europea que cuenta con preferencias arancelarias (Herrera, 2018).

El Acuerdo de Asociación entre la Comunidad Europea y Chile fue firmado a finales de 2002 cinco años después de que en Bruselas el 8 de diciembre de 1997 firmara la Unión Europea con México el Acuerdo de Asociación Económica, Concertación Política y Cooperación, el aguacate es un producto estratégico para la economía del sector primario, especialmente para México y pesar de existir un acuerdo que favorezca el comercio se observa que México no ha aprovechado los beneficios obtenidos por el acuerdo tanto como lo ha hecho Chile, esto es posible observarlo en las cifras de comercio se analizan en el presente artículo.

Los objetivos del artículo son comparar el éxito del intercambio comercial de la Unión Europea, Chile y México; revisar lo acordado a través del Acuerdo de Asociación Chile-Comunidad Europea con respecto al comercio de aguacate, para su posterior análisis y hacer conclusiones de la evolución del comercio generado a partir del acuerdo y comparar la experiencia comercial de Chile con la Unión Europea y la experiencia de México y con la Unión Europea en el comercio de aguacate.

\section{Revisión de literatura}

Existen algunos artículos relacionados de manera indirecta con el tema central de este artículo. Se enfocan en hacer análisis de los acuerdos comerciales existentes y retomados en este análisis, desde el punto de vista de la profundidad de las relaciones que generan entre las partes negociantes estos acuerdos; no se ha encontrado ningún artículo relacionado directamente aplicado al escrutinio del comercio de aguacate entre los socios comerciales entregados en esta propuesta.

Hay algunos artículos que han llamado atención y de los cuales se considera que será provechoso extraer ideas plasmadas en los mismos para dar soporte al planteamiento hecho aquí; desde el punto de vista del fomento de la creación de los acuerdos comerciales para el fomento de las relaciones entre los socios comerciales se menciona en el caso de Chile y la Unión Europea que "Con el objetivo de restaurar las relaciones de cooperación entre la Comunidad Europea y la República de Chile, que se basarían en el futuro en los valores democráticos y en el respeto a los derechos humanos, ambas Partes firmaron el primer Acuerdo Marco, el 20 de diciembre de 1990. El Acuerdo de tercera generación, abrió una nueva fase en las relaciones de la Comunidad con el país 
andino y sentó las bases para el desarrollo de una cooperación eminentemente económica entre ambas Partes (Altemir, 2004)".

Una idea planteada en este artículo es el hecho de uno de los sectores más favorecidos en el acuerdo de asociación entre México y la Unión Europea fue el sector agrícola; también está el supuesto que de igual manera Chile se vio beneficiado en este sector desde el punto de vista de la eliminación de aranceles, en un artículo se encontró referencia a este hecho y específicamente aborda el producto Palta (aguacate), Para el sector hortofrutícola la liberalización se escalonó en cuatro años para el 85 por 100 del sector, destacando las manzanas que se desgravan desde la entrada en vigor del acuerdo junto con las cebollas, espárragos frescos, pimentones, peras, ciruelas, paltas y frambuesas (Ana Fernández-Ardavín Martínez, 2006).

El tema central de este artículo que es la evolución del comercio de aguacate no está relacionado directamente en ninguna literatura consultada; la literatura consultada sirve para dar antecedentes de los supuestos planteados y ayudan a enriquecer el acervo de información que se ha tomado en cuenta para la realización del análisis.

Las principales consideraciones que se tuvieron para generar este análisis comercial es que México y Chile son dos de los países más importantes en el comercio internacional de aguacate desde el punto de vista de la oferta del producto. También que la Unión Europea es unos de los principales importadores del planeta esto es por lado de la oferta. El supuesto de un mercado clásico de oferta y demanda se puede asumir debido a que los acuerdos comerciales promovidos por la Unión Europea permite que ambos suplidores compitan en las mismas condiciones.

\section{Metodología}

El análisis del comercio de aguacate, implica la recopilación de información estadística expresada en series de tiempo con el fin de apreciar la evolución de las exportaciones de Chile a la Unión Europea, y las exportaciones de México a la Unión Europea de aguacate, en el periodo entre 2001 y 2016 lo cual representan 16 observaciones lo cual es significativo para analizar el comportamiento de la evolución de los volúmenes exportados de aguacate con origen en Chile y México hacia la Unión Europea.

Las series de tiempo se refiere a los datos recopilados con la misma regularidad, si un dato se registra con un periodo de tiempo exacto se debe de ser consistente al momento de la recopilación del siguiente dato, debe de ser registrado en forma periódica, es decir, al momento de recopilar el dato se debe de tomar en cuenta la temporalidad para formar una serie consistente; entre las series de tiempo más comunes que existen, en lo que ha temporalidad se refiere, están los datos recopilados diariamente, semanalmente, semestralmente y anualmente. 
El comercio de aguacate es cuantificado en toneladas, en el presente caso la medida que se utiliza son las toneladas exportadas registradas de manera anual, los datos se obtienen a través de los registros aduanales que se van sumando en el año en la medición, el hecho de que las bases de datos estén expresadas con la misma unidad de medida es conveniente para una comparación. Las bases de datos están expresadas con temporalidad anual, en la unidad de medida de toneladas y en un periodo homogéneo lo cual posibilita un análisis comparativo entre los dos competidores México y Chile tomando como referencia el mercado de la Unión Europea.

Uno de los indicadores que más resaltan al analizar diferentes proveedores es el precio al cual venden sus mercancías el cual por lo general es diferente para cada competidor, para este caso los datos presentados en la fuente TRADEMAP poseían toneladas exportadas y el valor de las exportaciones, debido a esto con estas dos estadísticas se debió de calcular el precio. Lo que se obtuvo fue precio por tonelada promedio anual lo que permite una comparación de precios obtenidos por los exportadores en el mercado europeo con la siguiente formula:

Ptpa $=V E / T E$

Dónde: Ptpa es el precio por tonelada promedio anual; Ve es el valor de las exportaciones; Te representan las toneladas exportadas.

Para el análisis pertinente se calcularon las tasas de crecimiento de los indicadores elegidos para el análisis de las exportaciones con la siguiente formula:

$T c=((V i-V f) / V i) * 100$,

Dónde: Tc es la tasa de crecimiento; Vi se refiere a valor inicial; Vf a valor final.

Lo cual permitió ligar el comportamiento de los volúmenes exportados con el precio, permite hacer una aproximación de tipo de demanda que existe el producto para determinar qué tipo de bien es para el mercado europeo, se parte bajo el supuesto clásico de que los mercados son regulados por el precio y que las cantidades demandadas deben de disminuir a un aumento del precio y aumentar frente a una disminución del precio, comportamiento que puede ser demostrado por la aplicación de una tasa de crecimiento debido a que toma como referencia valores anteriores y se observan disminuciones o aumentos de las cantidades expresadas en la serie de tiempo.

La proporción de marcado está calculada de manera que exprese que tanto representan las exportaciones de cada país vendedor en las importaciones de la Unión Europea, para el cálculo se toma en cuenta el volumen de las exportaciones de cada competidor y el volumen importado total de la Unión Europea con la siguiente formula:

$$
P m=V e / V i \text {, }
$$


Dónde: Pm es proporción de mercado; Ve es valor de las exportaciones; Vi es el valor de las importaciones totales.

A través de estos datos se calculó la proporción de mercado lo cual permite que sean comparables debido a que tienen el mismo mercado de destino.

\section{Resultados y Discusión \\ Del Acuerdo de Asociación México-Unión Europea}

El Acuerdo de Asociación Económica, Concertación Política y Cooperación (Acuerdo Global) que establece los objetivos y mecanismos para la liberación del comercio de bienes y de servicios, fue aprobado por el Parlamento Europeo el 6 de mayo de 1999 y por el Senado de México el 20 de marzo de 2000. El acuerdo fue adoptado por el Consejo Europeo el 28 de setiembre de 2000 y después de la notificación de las partes de la finalización de sus procedimientos legales internos necesarios para la entrada en vigor, comenzó a regir el $1^{\circ}$ de octubre de 2000.

Las relaciones de México con la Unión Europea han tenido varias etapas que se establecen en la siguiente cronología (Camara de diputados, $\mathrm{H}$. Congreso de la Unión, 2000):

- En 1960 se establece la Misión de México ante la Comunidad Económica Europea (CCE).

- El 15 de Julio de 1975, México y la CEE firman el Acuerdo de Cooperación Económica y Comercial.

- El 26 de Abril de 1991, México y la Unión Europea firman el Acuerdo Marco de cooperación.

- El 2 de Mayo de 1995 se emite la Declaración Conjunta Solemne entre México y la UE.

- El 8 de Diciembre de 1997 se crean dos instrumentos jurídicos entre la UE y México.

Luego de la entrada en vigor del Tratado de Libre Comercio de América del Norte (TLCAN) el $1^{\circ}$ de enero de 1994, la Unión Europea pretendió establecer relaciones comerciales privilegiadas con México con el objeto de mantener una posición estratégica dentro esta zona de libre comercio; no obstante, esta decisión se postergó por el temor de Francia de poner en riesgo la "Política Agrícola Común Europea" (PAC). A pesar de ello, entre noviembre de 1998 y noviembre de 1999 se concluyeron las discusiones sobre el contenido fundamental del primer acuerdo comercial entre la Unión Europea y un país de América Latina para crear una zona de libre comercio (Camara de diputados, H. Congreso de la Unión, 2000).

El aguacate tiene la fracción arancelaria 0804.40 el cual está especificado en la sexta sección del calendario de desgravación de México publicado en el Diario Oficial de la Federación; y partir de la entrada en vigor 
del Acuerdo de Asociación quedo con una tasa base de $20 \%$ de impuestos de importación desde Unión Europea; por lo cual se puede deducir que México en las negociaciones asumió una posición estratégica en el sentido que no desprotegió el mercado del aguacate al poner una barrera arancelaria.

\section{Del Acuerdo de Asociación Chile-Unión Europea}

Conociendo la postura de cada uno de los socios comerciales en materia de política agrícola se debe describir el acuerdo que existe entre ambos socios enfocado en materia agrícola y señalar si existe una preferencia para algún socio, o si en realidad la negociación en materia agrícola dejo a los competidores en igualdad de condiciones y si efectivamente se promueve el libre cambio de productos agrícolas y productos agrícolas transformados.

En el Acuerdo de Asociación Chile-Comunidad Europea el capítulo 1 especifica la eliminación de los derechos de aduana; el capítulo 1 a su vez tiene dos secciones la primera de disposiciones generales sobre la eliminación de los derechos aduanales y en la segunda sección se tratan tres grandes agrupaciones de productos y de la liberación de derechos de aduana, en específico la tercera, sección 2.3, agrupa "productos agrícolas y productos agrícolas transformados", esto en 5 artículos del acuerdo del 70-74.

En el artículo 70 se especifica el espectro del acuerdo, es decir el alcance o la envergadura de los componentes que va contener en concreto el ámbito de aplicación menciona que "se aplica a todos los productos agrícolas y productos agrícolas transformados cubiertos por la definición del Anexo I del Acuerdo sobre Agricultura de la OMC (Organización de los Estados Americanos, 2017)"'.

En el artículo 71 del Acuerdo de Asociación especifica un calendario de liberalización de derechos de aduanas de las importaciones originarias de Chile, existen cuatro momentos marcados de liberación comercial; para el primer momento se toma en cuenta la fecha que comenzó la vigencia del acuerdo que fue el primero de febrero de 2003, en el anexo 1 se especifican los productos que se comenzaran a liberalizar así como las cantidades de los mismos.

Dentro del mismo artículo 71 hay otros tres momentos marcados de liberación para cuatro, siete y diez años; con una disminución gradual cada año repartidos equitativamente hasta lograr el 100 por ciento de liberación a partir de la entrada en vigencia; para otros tres grupos de productos; el 1 de enero de 2007, el 1 de enero de 2010 y el 1 de enero de 2013, se deben de quedar liberados.

Por ejemplo los aguacates poseen la categoría "Year 0" lo que significa que la importación desde Chile estuvo liberado desde el día que entro en vigencia el Acuerdo de Asociación; como está especificado en la siguiente tabla. 
Tabla 1: Porcentajes de Porcentajes de reducción arancelaria anual originarias de Chile.

\begin{tabular}{|l|l|l|l|l|l|l|l|l|l|l|l|}
\hline Categoría & $\begin{array}{l}\text { Entrada } \\
\text { ln } \\
\text { vigor }\end{array}$ & 1.1 .04 & 1.1 .05 & 1.1 .06 & 1.1 .07 & 1.1 .08 & 1.1 .09 & 1.1 .10 & 1.1 .11 & 1.1 .12 & 1.1 .13 \\
\hline 0 años & $100.0 \%$ & & & & & & & & & & \\
\hline 4 años & $20.0 \%$ & $40.0 \%$ & $60.0 \%$ & $80.0 \%$ & $100.0 \%$ & & & & & & \\
\hline 7 años & $12.5 \%$ & $25.0 \%$ & $37.5 \%$ & $50.0 \%$ & $62.5 \%$ & $75.0 \%$ & $87.5 \%$ & $100.0 \%$ & & & \\
\hline 10 años & $9.0 \%$ & $18.0 \%$ & $27.0 \%$ & $36.0 \%$ & $45.0 \%$ & $54.0 \%$ & $63.0 \%$ & $72.0 \%$ & $81.0 \%$ & $90.0 \%$ & $100.0 \%$ \\
\hline
\end{tabular}

Fuente: Organización de los Estados Americanos, 2017. Sistema de información sobre

Comercio Exterior.

La liberación aduanal se da paulatinamente para los productos que provienen de Chile; de igual manera se hace para los productos que se importan desde Europa hacia Chile, pero en plazos distintos que en comparación a los plazos de los productos que van de Chile a Europa.

En el artículo 72 trata de los derechos de aduana sobre las importaciones de productos agrícolas y productos agrícolas transformados originarios de la comunidad; a diferencia de las provenientes de Chile solo posee tres categorías "Year 0", "Year 5" y "Year 10" que a su vez marcan los plazos en los que se acordó hacer la liberación del derecho aduanal y las fechas fueron el día de la entrada en vigor, el 1 enero de 2008 y el 1 de enero de 2013 para cada una de las categorías; en el mismo artículo 72 del Acuerdo de Asociación está incluida una tabla en la que especifica la transición.

Tabla 2: Porcentajes de reducción arancelaria anual originarias de la Comunidad Europea.

\begin{tabular}{|l|l|l|l|l|l|l|l|l|l|l|l|}
\hline Categoría & $\begin{array}{l}\text { Entrada } \\
\text { en vigor }\end{array}$ & 1.1 .04 & 1.1 .05 & 1.1 .06 & 1.1 .07 & 1.1 .08 & 1.1 .09 & 1.1 .10 & 1.1 .11 & 1.1 .12 & 1.1 .13 \\
\hline 0 años & $100.0 \%$ & & & & & & & & & & \\
\hline 5 años & $16.7 \%$ & $33.3 \%$ & $50.0 \%$ & $66.6 \%$ & $83.3 \%$ & $100.0 \%$ & & & & & \\
\hline 10 años & $9.0 \%$ & $18.0 \%$ & $27.0 \%$ & $36.0 \%$ & $45.0 \%$ & $54.0 \%$ & $63.0 \%$ & $72.0 \%$ & $81.0 \%$ & $90.0 \%$ & $100.0 \%$ \\
\hline
\end{tabular}

Fuente: Organización de los Estados Americanos, 2017. Sistema de Información sobre Comercio Exterior.

En el caso del aguacate están en mismo término poseen la categoría "Year 0"; ambos socios comerciales en términos de este producto decidieron eliminar las barreras arancelarias al mismo tiempo y desde el momento de entrada en vigor del Acuerdo de Asociación; por ende la ventaja se basara en la ventaja de producción que tenga uno sobre el otro.

El artículo 73 de Acuerdo es una cláusula de emergencia de suspensión del proceso de reducción arancelaria ya a su vez aumentar el derecho de aduana siempre y cuando no exceda dos limites el primero que supere el derecho de la nación más favorecida y a su vez que no transgreda la reducción gradual del derecho aduanal; y esto se debió hacer específicamente cuando "dada la particular sensibilidad de los mercados agrícolas, un producto originario de una parte se importa en la otra parte en cantidades o en condiciones que causen o amenacen con causar un perjuicio o perturbación importante en los mercados 
de productos similares o directamente competitivos de la otra parte, esta última podrá adoptar las medidas apropiadas en las condiciones y con arreglo a los procedimientos establecidos (Organización de los Estados Americanos, 2017)".

Finalmente en el artículo 74 del Acuerdo de Asociación se trata una cláusula evolutiva es decir un seguimiento de lo acordado; ambas partes se comprometen a evaluar la evolución del comercio de los productos agrícolas y agrícolas transformados tomando en cuenta la sensibilidad del mercado y se evaluara si hay reciprocidad adecuada en los términos de comercio y la evaluación se acordó que se debería hacer producto por producto.

\section{Comercio entre México y la Unión Europea}

El aguacate representa para México uno de los más importantes productos de exportación de los productos agrícolas; en el mundo el aguacate mexicano es muy apreciado por los consumidores del fruto, en particular por los europeos. La demanda permite que exista comercio de aguacate entre la Unión Europea y México el Acuerdo de Asociación ha permitido que exista un crecimiento significativo en las exportaciones de mexicanas a la Unión Europea.

Tabla 3. Evolución de las exportaciones de aguacate de México de 2001 a 2016.

\begin{tabular}{|l|l|l|l|l|l|}
\hline Años & $\begin{array}{l}\text { Dólares } \\
\text { Americanos }\end{array}$ & Toneladas Exportadas & Años & $\begin{array}{l}\text { Dólares } \\
\text { Americanos }\end{array}$ & Toneladas Exportadas \\
\hline 2001 & 14093000 & 12777 & 2009 & 17669000 & 10293 \\
\hline 2002 & 13394000 & 11944 & 2010 & 12259000 & 7126 \\
\hline 2003 & 37061000 & 23275 & 2011 & 5446000 & 2702 \\
\hline 2004 & 20576000 & 14068 & 2012 & 8217000 & 5068 \\
\hline 2005 & 26946000 & 16958 & 2013 & 15861000 & 8430 \\
\hline 2006 & 25198000 & 15570 & 2014 & 22492000 & 11152 \\
\hline 2007 & 22621000 & 11512 & 2015 & 45991000 & 26180 \\
\hline 2008 & 22759000 & 11713 & 2016 & 118433000 & 52887 \\
\hline
\end{tabular}

Fuente: Elaboración propia con datos de TRADEMAP.

En cifras se puede apreciar que si solo se toman en cuenta los años extremos es decir 2001 y 2016 existe un crecimiento de casi cinco veces más en el volumen exportado, pero esta observación solo sirve para dar una acercamiento general; es destacable el hecho que en los años en los que estuvo latente la crisis financiera internacional el comercio disminuyo y los volúmenes intercambiados tuvieron un comportamiento muy volátil; lo cual no permite observar una tendencia clara en el comportamiento de los volúmenes de exportación hacia la Unión Europea desde México.

En la siguiente grafica se puede ver la tendencia de la evolución de las exportaciones, aunque no está acentuada debido a la abrupta disminución de 
las exportaciones en el periodo de la crisis financiera internacional y que comenzaron a recuperar su crecimiento a partir de 2012.

Grafica 1: Toneladas Exportadas de aguacate mexicano hacia la Unión Europea de 2001 a 2016.

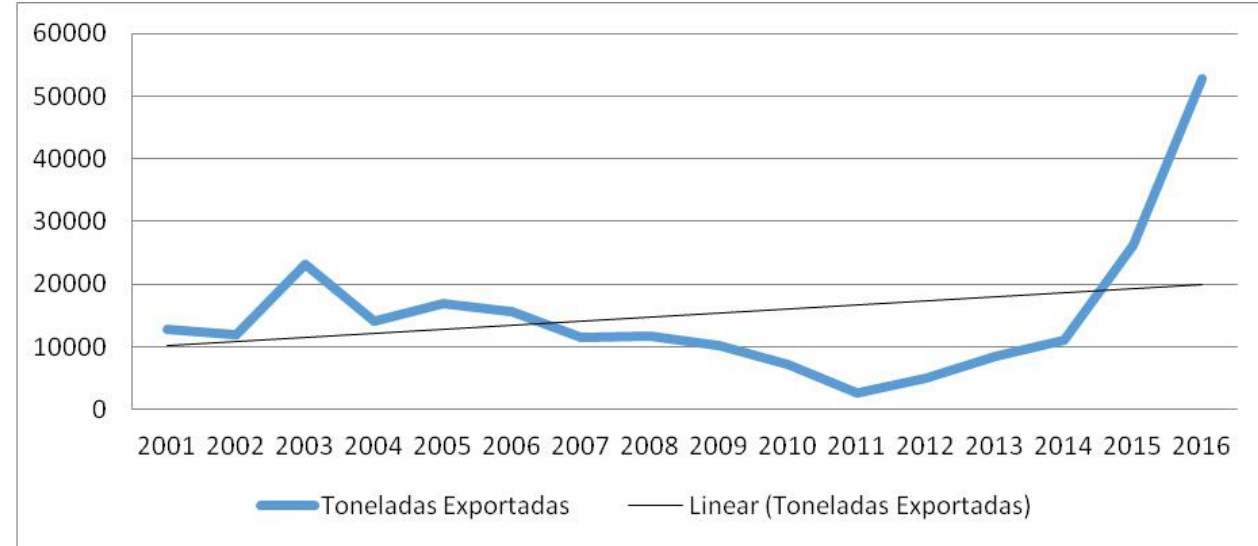

Fuente: Elaboración propia con datos de TRADEMAP

La expresión gráfica muestra el comportamiento de los volúmenes exportados hacia la Unión Europea desde México, en general se muestra una tendencia creciente en el volumen de las exportaciones en el periodo observado; en el periodo de 2001 a 2011 las exportaciones son en general decrecientes con excepción de los años 2003, 2005 y 2008.

En 2012 hubo un aumento significativo con un $87.56 \%$ con respecto al año anterior, y partir de entonces hasta el año 2016 se han mantenido con una tasa de crecimiento promedio de $84.59 \%$, siendo el año de menor crecimiento el 2014 con $32.29 \%$ y el año de mayor crecimiento fue 2015 con $134.76 \%$ con respecto al año anterior inmediato.

A partir de año de entrada en vigor del Acuerdo de Asociación se observa un crecimiento moderado en las tasas de crecimiento de las exportaciones a pesar de la caída en la mayoría de los años del estudio, a partir del año 2012 es cuando el aguacate importado desde México hacia la Unión Europea ha conquistado el mercado Europeo y se hace notar aprovechado el Acuerdo de Asociación.

\section{Evolución de los precios de las exportaciones de aguacate mexicano a la Unión Europea}

Los precios promedios por tonelada están expresados en dólares americanos, los precios en el transcurso del periodo observado desde el inicio hasta el final se aprecia que el precio se duplico al final de tiempo transcurrido existen fluctuaciones negativas y positivas en los precios promedios. 
Tabla 4. Precios anuales de las exportaciones de aguacate mexicano a la Unión Europea 2001 a 2016.

\begin{tabular}{|l|l|l|l|}
\hline Años & $\begin{array}{l}\text { Precio promedio por } \\
\text { tonelada en dólares } \\
\text { americanos }\end{array}$ & Años & $\begin{array}{l}\text { Precio promedio por tonelada en } \\
\text { dólares americanos }\end{array}$ \\
\hline 2001 & 1103.0 & 2009 & 1716.6 \\
\hline 2002 & 1121.4 & 2010 & 1720.3 \\
\hline 2003 & 1592.3 & 2011 & 2015.5 \\
\hline 2004 & 1462.6 & 2012 & 1621.3 \\
\hline 2005 & 1589.0 & 2013 & 1881.5 \\
\hline 2006 & 1618.4 & 2014 & 2016.9 \\
\hline 2007 & 1965.0 & 2015 & 1756.7 \\
\hline 2008 & 1943.1 & 2016 & 2239.4 \\
\hline
\end{tabular}

Fuente: Elaboración propia con datos de TRADEMAP.

En los años 2002, 2003, 2005, 2006, 2007, 2010, 2011, 2013, 2014 y 2016 las fluctuaciones de los precios fueron positivas con respecto al año anterior inmediato.

La teoría de la oferta y demanda busca explicar cómo se llega al equilibrio de un mercado a través del precio, a mayor precio menor demanda y viceversa, es por ello que las tasa de crecimiento deberían demostrar una tendencia totalmente contraria si se tratase de un bien normal, en la siguiente ilustración se muestran la tasas de crecimiento del comportamiento de los volúmenes de las exportaciones y las tasas de crecimiento de los precios de las exportaciones en cada año observado.

Grafica 2: Relación de las tasas de crecimiento de los precios promedio y de los volúmenes

comerciados de aguacate mexicano hacia la Unión Europea de 2002 a 2016.

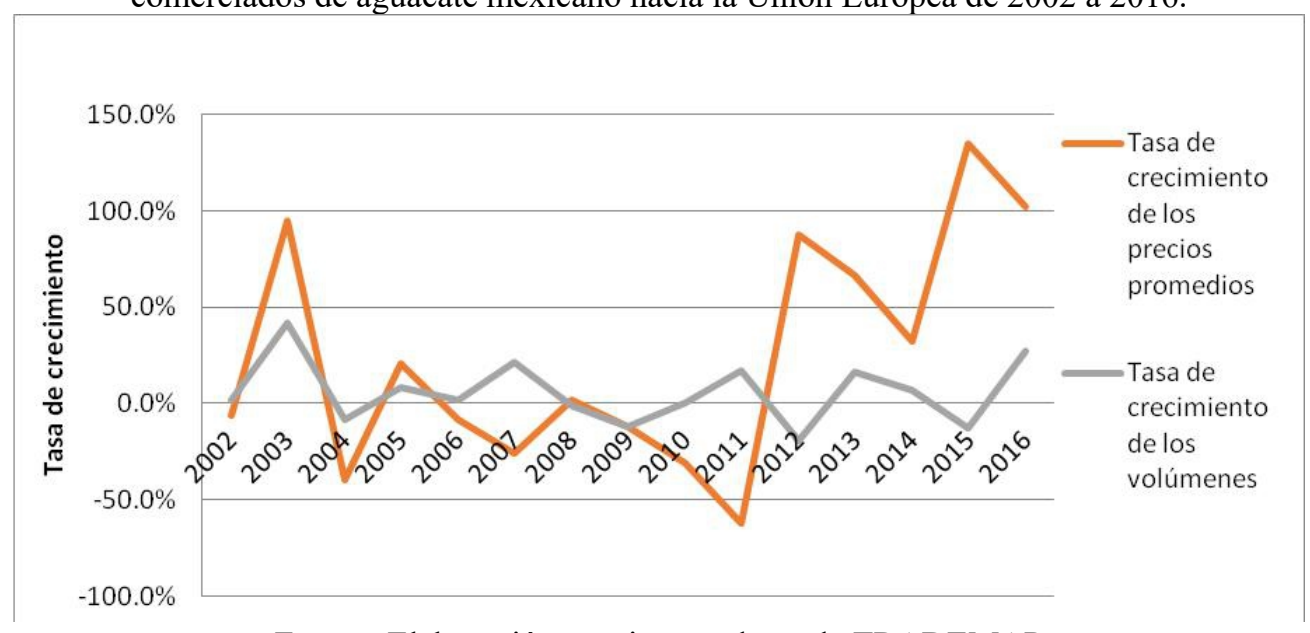

Fuente: Elaboración propia con datos de TRADEMAP

Las tasas de crecimiento se comportaron de la misma manera cuando aumentaron las cantidades exportadas los precios también aumentaron en los 
años 2003, 2005, 2013, 2014 y 2016; en 2004 y 2009 cuando disminuyó la tasa de crecimiento de los volúmenes exportado también disminuyo la tasa de crecimiento de los precios conseguidos en el mercado. El comportamiento de los precios del mercado internacional del aguacate no corresponde al comportamiento de un mercado clásico de oferta y demanda en los siete años señalados.

En la mayoría de los años del periodo de análisis 2002, 2006, 2007, 2008, 2010, 2011, 2012 y 2015 si se observó un comportamiento contrario de las tasas de interés de las cantidades y precios que se observan en las exportaciones de aguacates de México hacia la Unión Europea, lo cual significa que el aguacate posee las características de un bien normal; el comercio de aguacate no genera un mercado habitual de oferta y demanda, es más bien fluctuante e impredecible en su comportamiento.

Comercio entre Chile y la Unión Europea.

El mercado europeo es demandante y exportador neto de aguacate, por lo cual Chile ha aprovechado el Asociación existente con la Unión Europea, es por esta razón que es conveniente revisar el comportamiento de los valores de las exportaciones y los volúmenes para hacer conclusiones sobre la evolución del comercio; en 2002 se firmó el Acuerdo de Asociación y en febrero de 2003 la importación de aguacates desde Chile hacia la Unión Europea estuvo libre de aranceles en un $100 \%$. Las exportaciones analizadas serán a partir del 2001 para la comparación que se pretende.

Tabla 5. Evolución de las exportaciones de aguacate de Chile a la Unión Europea de 2001 a 2016.

\begin{tabular}{|l|l|l|l|l|l|}
\hline Años & $\begin{array}{l}\text { Dólares } \\
\text { Americanos }\end{array}$ & Toneladas Exportadas & Años & $\begin{array}{l}\text { Dólares } \\
\text { Americanos }\end{array}$ & Toneladas Exportadas \\
\hline 2001 & 469000 & 374 & 2009 & 70662000 & 43714 \\
\hline 2002 & 1777000 & 1299 & 2010 & 63694000 & 33562 \\
\hline 2003 & 6739000 & 4032 & 2011 & 54002000 & 27223 \\
\hline 2004 & 14410000 & 11098 & 2012 & 75585000 & 40237 \\
\hline 2005 & 24169000 & 18188 & 2013 & 112187000 & 52723 \\
\hline 2006 & 44614000 & 35386 & 2014 & 114084000 & 54522 \\
\hline 2007 & 51229000 & 30398 & 2015 & 144778000 & 63361 \\
\hline 2008 & 29550000 & 17266 & 2016 & 209922000 & 90723 \\
\hline
\end{tabular}

Fuente. Elaboración propia con datos de TRADEMAP.

Si se compara el año inicial hasta el último año es evidente un aumento abrupto de las exportaciones chilenas hacia la Unión Europea; para una mejor apreciación de la evolución es necesario la representación gráfica de las exportaciones a través de los años incluidos en la tabla se expresaran las toneladas exportadas. 
Grafica 3: Toneladas Exportadas de aguacate chileno hacia la Unión Europea de 2001 a 2016.

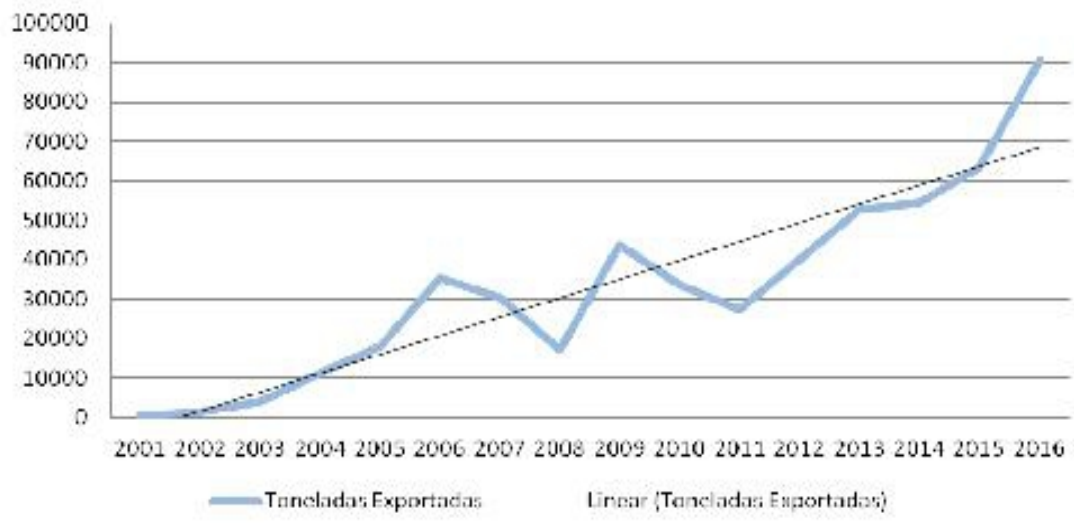

Fuente: Elaboración propia con datos de TRADEMAP.

La expresión gráfica muestra el comportamiento de los volúmenes exportados hacia la Unión Europea desde Chile, en general se muestra una tendencia creciente en el volumen de las exportaciones en el periodo observado.

En los años 2007 y 2008 se observa una reducción en los volúmenes exportados de aguacate, en términos porcentuales hubo una reducción de $14.1 \%$ y $43.2 \%$ con respecto a cada año anterior. En 2009 hubo un aumento muy significativo con un $153 \%$ con respecto al año anterior y que supera el volumen que se comercio en 2006 que fue antes de que comenzara la reducción de los volúmenes comerciados.

Nuevamente en los años 2010 y 2011 existió una reducción en los volúmenes exportados de Chile a la Unión Europea, en términos porcentuales las reducciones fueron de $23.22 \%$ y $18.89 \%$ con respecto al año anterior inmediato. En el año 2012 las exportaciones repuntaron, y partir de entonces hasta el año 2016 se han mantenido con una tasa de crecimiento promedio de $28.33 \%$, siendo el año de menor crecimiento el 2014 con $3.41 \%$ y el año de mayor crecimiento fue 2012 con $47.81 \%$.

En conclusión a partir de año de entrada en vigor del Acuerdo de Asociación se observa un crecimiento abrupto en las exportaciones a pesar de la caída de los años 2007, 2008, 2010 y 2011, no se exporto menos que la cantidad comerciada en el año 2002, que fue el último año que no se contó con el Acuerdo de Asociación entre Chile y la Unión Europea; es por ello que se afirma que para Chile ha sido benéfico la formalización de la relación comercial con la Unión Europea en términos del comercio de aguacate.

Evolución de los precios de las exportaciones de aguacate de Chile a la Unión Europea 
Tabla 6. Precios anuales de las exportaciones de aguacate de Chile a la Unión Europea de 2001 a 2016.

\begin{tabular}{|l|l|l|l|}
\hline Años & $\begin{array}{l}\text { Precio promedio por tonelada en dólares } \\
\text { americanos }\end{array}$ & Años & $\begin{array}{l}\text { Precio promedio por tonelada en dólares } \\
\text { americanos }\end{array}$ \\
\hline 2001 & 1254.0 & 2009 & 1616.5 \\
\hline 2002 & 1368.0 & 2010 & 1897.8 \\
\hline 2003 & 1671.4 & 2011 & 1983.7 \\
\hline 2004 & 1298.4 & 2012 & 1878.5 \\
\hline 2005 & 1328.8 & 2013 & 2127.9 \\
\hline 2006 & 1260.8 & 2014 & 2092.4 \\
\hline 2007 & 1685.3 & 2015 & 2285.0 \\
\hline 2008 & 1711.5 & 2016 & 2313.9 \\
\hline
\end{tabular}

Fuente. Elaboración propia con datos de TRADEMAP.

Los precios promedios por tonelada están expresados en dólares americanos, los precios en el transcurso del periodo observado desde el inicio hasta el final se aprecia que el precio casi duplico al final de tiempo transcurrido existieron fluctuaciones negativas y positivas en los precios del periodo observado; en los años 2002, 2003, 2005, 2007, 2008, 2010, 2011, 2013, 2015 y 2016 las fluctuaciones de los precios fueron positivas con respecto al año anterior inmediato.

El comportamiento general en los precios de los productos debe de ser a la alza para que los productores de los bienes estén incentivados en la producción de los mismos, si existe una disminución en los precios es muy lógico que los productores se sientan desincentivados a seguir produciendo. Los precios además de equilibrar los mercados internacionales pueden frenar o acelerar la producción existente de aguacate, o bien los productores buscan otros mercados con mejores precios.

Grafica 4: Relación de las tasas de crecimiento de los precios promedio y de los volúmenes comerciados de aguacate chileno hacia la Unión Europea de 2002 a 2016.

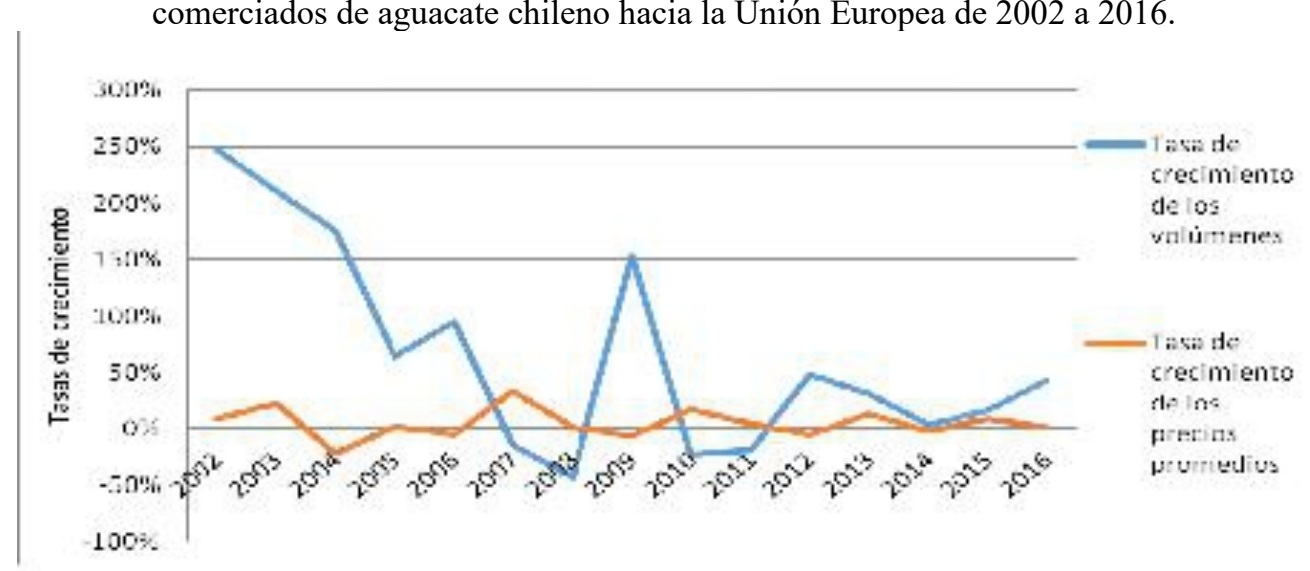

Fuente. Elaboración propia con datos de TRADEMAP. 
En algunos años las tasas de crecimiento se comportaron de la misma manera cuando aumentaron las cantidades exportadas los precios también aumentaron; esto se observó en los años 2002, 2003, 2005, 2013, 2015 y 2016; en ninguno de los años disminuyó la tasa de crecimiento de los volúmenes exportados y al mismo tiempo disminuyo la tasa de crecimiento de los precios conseguidos en el mercado. En estos años el comportamiento de los precios del mercado internacional del aguacate no conllevó a una disminución del crecimiento de las exportaciones, por esta razón no corresponde al comportamiento de un mercado clásico de oferta y demanda.

En la mayoría de los años del periodo de análisis 2004, 2006, 2007, 2008, 2009, 2010, 2011, 2012 y 2014 si se observó un comportamiento contrario de las tasas de crecimiento de las cantidades y precios que se observan en las exportaciones de aguacates de Chile hacia la Unión Europea, lo cual significa que el aguacate posee las características de un bien normal; el comercio de aguacate no genera un mercado habitual de oferta y demanda; la relación de las tasas de interés se vuelve predecible ya que la mayoría de los años el mercado se comporta como un mercado clásico de oferta y demanda.

\section{Comparación entre México y Chile de la proporción de mercado en las importaciones totales de la Unión Europea}

El socio comercial en común que tienen Chile y México en el comercio de aguacate es la Unión Europea y es en este mercado donde se debe comparar el comercio aguacate para determinar para quien es más importante el mercado europeo y quien ha aprovechado mejor el Acuerdo de Asociación. Para el análisis es deseable ver cuál es la proporción del total de exportaciones de cada país hacia la Unión Europea, para así determinar para cual país el mercado europeo tiene mayor importancia en el comercio de aguacate.

Grafica 4: Proporción de las exportaciones de Chile Y México de las importaciones de aguacate de la Unión Europea de 2001 a 2016.



Fuente. Elaboración propia con datos de TRADEMAP. 
En esta grafica se aprecia que tanto representan las exportaciones de cada uno de los socios comerciales en la demanda total de la Unión Europea; en general el hecho más apreciable la conquista de la proporción del mercado europeo de parte de Chile en el año 2001 las exportaciones de Chile hacia la Unión Europea fueron de $0.2 \%$ y a través de los años fue aumentando, llegando en su punto más alto en el 2009 año en el que llego a representar el 15.2\% de las importaciones totales de la Unión Europea; es decir en nueve años se apropió del 15\% de la demanda Europea y si se toma en cuenta la entrada en vigor del Acuerdo de Asociación a partir de 2013 es decir en siete años llego a cubrir la demanda de las importaciones de aguacate de la Unión Europea. A partir del 2010 la proporción de las importaciones cubiertas por las exportaciones de Chile decrece, a partir del 2011 recupera y comienza a crecer, termina en 2016 con 12.1\% de las importaciones totales de la Unión Europea.

Con respecto a la proporción de las importaciones de la Unión Europea cubiertas por las exportaciones México en 2001 poseía un 6.9\% de la proporción de las importaciones de la Unión Europea, en 2003 fue el año con mayor cantidad de mercado cubierto por México con un $12.7 \%$ de la proporción de las importaciones, a partir del año siguiente comienza a decrecer la proporción de merado satisfecha por México, mismo año en el que el Acuerdo de Asociación entre Chile y la Unión Europea entra en vigor, la explicación de la caída en 2004 del aguacate mexicano en la Unión Europea podría estar explicado por el precio al cual ofertaban en el mercado el cual fue de 1462.61 dólares americanos por tonelada, mientras que Chile aumentaba su cuota de mercado con un precio menor de 1298.43 dólares americanos por tonelada.

En fue el año 2011 fue cuando México tuvo la proporción de mercado más baja registrada en el periodo de estudio, solo represento un $0.8 \%$ del mercado de la Unión Europea, a partir de ese entonces es que comienza a crecer llegando a 2016 a un 7\% de la proporción del mercado europeo, proporción similar pero ligeramente superior al año de entrada en vigor del Acuerdo de Asociación entre México y la Unión Europea la cuota de mercado de México se mantiene estancada y no presenta una tendencia creciente tan clara como la que presenta Chile.

\section{Conclusion}

La dinámica económica de los agentes comerciales los lleva a formalizar acuerdos comerciales; al tomar auge el comercio entre los agentes comerciales nace la necesidad de generar acuerdos que potencien el comercio. Los acuerdos comerciales son el principio de relaciones entre naciones que pueden llegar a desencadenar cooperación en sectores más allá del comercio. 
El Acuerdo de Asociación entre Chile y la Unión Europea está enfocado en la eliminación de los derechos de aduana, lo cual se da a partir de febrero de 2003 con la entrada en vigor del acuerdo; se analizó el aguacate como producto y su normatividad dentro del Acuerdo de Asociación los aguacates estuvieron liberados desde el momento de la entrada en vigor, referente a la importación desde Chile. Los aguacates que vallan de la Unión Europea hacia Chile posee la misma clasificación es decir que estuvo libre de derechos aduanales desde el primer día de la entrada en vigor del acuerdo, es decir, que ambos socios comerciales en términos del aguacate tuvieron una liberalización igualitaria.

Cada país debe de tomar posturas para negociar con otros; para México debe ser de suma importancia la experiencia chilena al momento de renegociar el acuerdo comercial existente con la Unión Europea; debe de fijar los objetivos que se pretendan alcanzar en una negociación futura en materia agrícola protegiendo e incentivando a los productores mexicanos con instrumentos de política publican que permitan el desarrollo del campo mexicano.

En el comercio entre México y la Unión Europea en el periodo de estudio entre 2001 y 2016 existe un crecimiento de casi cinco veces más en el volumen exportado, pero el comportamiento no refleja una tendencia clara; en general se muestra una tendencia creciente en el volumen de las exportaciones en el periodo observado. En el periodo de 2001 a 2011 las exportaciones fueron en general decrecientes, en 2012 la tendencia comenzó a ser creciente hasta 2016, este periodo de cinco años propicio la ten decencia general creciente.

En general los precios del aguacate mexicano hacia la Unión Europea se duplicaron en el periodo de estudio de 2001 a 2016, en la mayoría de los años las fluctuaciones son positivas con respecto al año anterior. En siete de los años del periodo de estudio cuando aumento el precio aumentaron también los volúmenes exportados, pero en la mayoría de los años de estudio cuando aumentaron las exportaciones disminuyo el precio, es decir se comportó como en general como un bien normal.

En el periodo de estudio las exportaciones chilenas hacia la Unión Europea. Existe una tendencia claramente creciente en las toneladas exportadas, en los años de mayor reducción de la Economía mundial a causa de la crisis financiera internacional 2007 y 2008, existieron reducciones significativas en las exportaciones de aguacate de Chile; en el periodo de 20122016 el crecimiento es más claro aún, debido a que hubo aumento en todos estos años.

Con respecto a los precios de los aguacates exportados de Chile hacia la Unión Europea, se experimentaron incrementos de los en diez años de los observados; en la mayoría de los años se observó un comportamiento contrario de las tasas de crecimiento de las cantidades y precios que se observan en las 
exportaciones, lo cual confirma el hecho que para el mercado europeo en general en el periodo observado el aguacate se comporta como un bien normal.

Al comparar las cuotas de mercado de aguacate de Chile y México en las Importaciones totales de la Unión Europea, el hecho más notable es que Chile ha crecido en el mercado europeo en 2001 las exportaciones chilenas a la Unión Europea representaba menos del 1\% de las importaciones totales de la Unión Europea, 2016 represento 12.1\% y en el año de mayor proporción 2015 llego a representar 15.2\%, el 1 Acuerdo de Asociación para Chile ha propiciado una evolución significativa de en el mercado europeo de aguacate.

En el caso de México se observa en general ha mantenido una cuota cercana al 7\% del mercado de aguacate en Europa; el año en que se registraron menos exportaciones mexicanas fue el año 2011 con menos de un punto porcentual, a partir del año siguiente comienza el crecimiento de las exportaciones llegando en 2016 al 7\% del total de las importaciones de la Unión Europea. El Acuerdo de Asociación ha servido para México para mantener su proporción de mercado, más no para aumentarla significativamente como el caso de Chile.

\section{References:}

\section{Literatura Citada}

1. Altemir, A. B. (01 de Enero de 2004). Google Academico. Recuperado el 24 de Agosto de 2018, de Google Academico: https://dadun.unav.edu/bitstream/10171/22180/1/ADI_XX_2004_02. pdf

2. Ana Fernández-Ardavín Martínez, I. H. (24 de Enero de 2006). Revista ICE. Recuperado el 24 de Agosto de 2018, de Revista ICE: http://www.revistasice.com/CachePDF/ICE_825_249-Camara de diputados, H. Congreso de la Unión. (1 de Febrero de 2000). Centro de estudios de las finanzas publicas. Recuperado el 2017 de 11 de 07, de Centro de estudios de las finanzas publicas: http://www.cefp.gob.mx/intr/edocumentos/pdf/cefp/cefp0062000.pdf

3. Herrera, M. (24 de Enero de 2018). El Sol de México. Obtenido de El Sol de México: https://www.elsoldemexico.com.mx/analisis/modernizacion-deltlcuem-726301.html

4. Organización de los Estados Americanos. (2017). Sistema de Informacion sobre Comercio Exterior. Recuperado el 17 de Junio de 2017, de http://www.sice.oas.org/Trade/ChiEU_s/ChEUin_s.asp

\section{Literatura consultada}

5. Cano, J. E. (1 de julio de 2014). Redalyc. Recuperado el 2017 de septiembre de 6, de http://www.redalyc.org/pdf/141/14131676004.pdf 
6. Comisión Europea. (Diciembre de 2016). Unión Europea. Recuperado el 7 de Marzo de 2017, de https://bookshop.europa.eu/es/agriculturepbNA0216625/

7. Delegacion de la Unión Europea en México. (2016). Delegacion de la Unión Europea en México. Recuperado el 21 de Octubre de 2016, de http://eeas.europa.eu/delegations/mexico/eu_mexico/political_relation s/political_relations_framework/index_es.htm

8. Fernández-Ardavín Martínez, A., \& Hurtado Ocaña, I. (SeptiembreOctubre de 2005). Revistas ICE. Recuperado el 3 de Marzo de 2017, de http://www.revistasice.com/cachepdf/ice_825_249269_f4ebd9af492375c1ee9ae2570f89e51.pdf

9. Forbes Staff. (10 de Julio de 2014). Forbes México. Recuperado el 18 de Octubre de 2016, de http://www.forbes.com.mx/tratados-de-librecomercio-mucha-politica-y-poca-riqueza/\#gs.z00Bw0Q

10. Ministerio de Agricultura. (2017). MINAGRI. Recuperado el 07 de Junio de 2017 , de http://www.minagri.gob.cl/institucion/institucional/quienes-somos/

11. Ministerio de Agricultura. (2017). MINAGRI. Recuperado el 7 de Junio de 2017 , de http://www.minagri.gob.cl/institucion/institucional/lineamientos_estra tegicos/

12. Montemayor Marin, M. C., Bijarro Hernandez, F., \& Estrada Bellman, P. C. (2007). POLITICAS Y GESTION PUBLICA PARA EL ESTUDIO MUNICIPAL: OPTICA ACADEMICA. CD. VICTORIA: D.R. Universidad Autónoma de Tamaulipas.

13. Parlamento Europeo. (1 de Junio de 2017). Parlamento Europeo. Recuperado el 19 de Agosto de 2017, de http://www.europarl.europa.eu/atyourservice/es/displayFtu.html?ftuId =FTU_6.2.2.html

14. Peña, G. B. (1968). LA POLITICA ECONOMICA PARA LA AGRICULTURA (PRIMERA ed.). México D.F: EDITORIAL AGRONOMICA MEXICANA S.A.

15. Portilla, B. (Febrero de 2000). Repositorio digital cepal. Recuperado el 6 de Junio de 2017, de http://repositorio.cepal.org/handle/11362/4674

16. SAGARPA. (1 de Marzo de 2010). Sagarpa. Obtenido de Sagarpa: http://www.sagarpa.gob.mx/programas2/evaluacionesExternas/Lists/ Otros\%20Estudios/Attachments/34/analisis_de_\%20instrumentos_pol itica_agropecuaria_rural_y_pesquera_en_Mexico\%20(Vol.\%20I).pdf

17. Wilson Center. (01 de Enero de 2017). Wilson Center. Obtenido de Wilson Center:

https://www.wilsoncenter.org/sites/default/files/Subsidios_Cap_1_Fo $\mathrm{x} \% 20$ and $\% 20$ Haight.pdf 